\title{
OryzaPG-DB: Rice Proteome Database based on Shotgun Proteogenomics
}

\author{
Mohamed Helmy ${ }^{1,2}$, Masaru Tomita ${ }^{1}$ and Yasushi Ishihama ${ }^{1,3^{*}}$
}

\begin{abstract}
Background: Proteogenomics aims to utilize experimental proteome information for refinement of genome annotation. Since mass spectrometry-based shotgun proteomics approaches provide large-scale peptide sequencing data with high throughput, a data repository for shotgun proteogenomics would represent a valuable source of gene expression evidence at the translational level for genome re-annotation.

Description: Here, we present OryzaPG-DB, a rice proteome database based on shotgun proteogenomics, which incorporates the genomic features of experimental shotgun proteomics data. This version of the database was created from the results of 27 nanoLC-MS/MS runs on a hybrid ion trap-orbitrap mass spectrometer, which offers high accuracy for analyzing tryptic digests from undifferentiated cultured rice cells. Peptides were identified by searching the product ion spectra against the protein, cDNA, transcript and genome databases from Michigan State University, and were mapped to the rice genome. Approximately 3200 genes were covered by these peptides and 40 of them contained novel genomic features. Users can search, download or navigate the database per chromosome, gene, protein, CDNA or transcript and download the updated annotations in standard GFF3 format, with visualization in PNG format. In addition, the database scheme of OryzaPG was designed to be generic and can be reused to host similar proteogenomic information for other species. OryzaPG is the first proteogenomics-based database of the rice proteome, providing peptide-based expression profiles, together with the corresponding genomic origin, including the annotation of novelty for each peptide.
\end{abstract}

Conclusions: The OryzaPG database was constructed and is freely available at http://oryzapg.iab.keio.ac.jp/.

\section{Background}

Among high-throughput experimental methods, genome sequencing represents a turning point in the understanding of biological systems. Nevertheless, the biological significance of the sequenced genome cannot be understood unless the protein-coding genes and their products are accurately identified. Thus, genome annotation has become major issue [1-3]. Genome annotation is the process of gene structure and function determination, and it usually takes place after genome sequencing and before data deposition in a database or databank $[2,4,5]$.

In typical genome annotation work, experimental and computational methods are integrated to analyze the huge volume of sequence data $[2,4,6,7]$. Thus, genome

\footnotetext{
* Correspondence: y-ishi@ttck.keio.ac.jp

'Institute for Advanced Biosciences, Keio University, 403-1 Daihoji, Tsuruoka, Yamagata 997-0017, Japan

Full list of author information is available at the end of the article
}

annotation is highly dependent on the expression evidence, usually transcriptional, provided by experiments and the algorithms implemented in the computational tools [8]. Consequently, the annotation process suffers from several limitations. For instance, most of the sequenced genomes lack rich transcriptional evidence, e.g., a full-length cDNA library. Even when such information is available, evidence of expression at the transcriptional level does not necessarily imply translation into a protein $[8,9]$. Therefore, annotation is highly reliant on de novo annotations of protein-coding genes performed using gene prediction programs $[2,4,8]$.

On the other hand, gene/protein prediction tools have proven their usefulness and utility in the annotation process. However, the prediction accuracy varies from one tool/algorithm to another and from one organism to another, depending on the genome complexity $[2,8,10,11]$. For instance, in the human and Arabidopsis genomes, the prediction accuracy amounted to $50 \%$ and 
$\sim 66 \%$, respectively, indicating the need for better identification and validation methods [11,12].

Mass spectrometry-based proteomics, as an experimental approach to measure proteins, can provide translation-level expression evidence for the predicted protein-coding genes; this is the so-called proteogenomics approach of using large-scale proteome data in genome annotation refinement $[3,8,13,14]$. This approach seems the best option for identification and validation of protein-coding genes, or at least a significant portion of them, in an independent and unambiguous way. This can be achieved by detecting the naturally occurring proteins (proteomics) and systematically mapping them back to the genome sequence (genomics) $[3,8,13,14]$. In addition to validating predicted gene models at the translation level $[15,16]$, proteogenomics has other useful applications, such as finding new gene models [7], determination of protein start and termination sites [17], finding and verifying splice isoforms at the protein level [18] and verification of hypothetical and putative genes/proteins $[17,19]$. The results of proteogenomic studies are usually made freely available via specialized databases such as AgBase [20] or are included in databases developed particularly to host data from specific projects, such as the AtProteome database developed to host the Arabidopsis proteogenomics data [21]. Overall, proteogenomics represents a promising approach for application to both completed and newly sequenced genomes.

Rice (Oryza sativa) is one of the most important food crops; almost half of the world's population is estimated to rely totally or partially on it. Moreover, rice considered a model organism because of its relatively small genome (12 chromosomes and $2370 \mathrm{Mbp}$ ) [22,23]. The whole genome sequence and annotation have been published and updated several times ( 5 builds for the genome and 6 builds for the annotation to date) [24-26]. However, there has been little attempt to include proteome information in the genome-wide annotation, except for the work of Itoh and colleagues, who used rice proteome data, available through the rice proteome database [27], to confirm 834 ORFs [25]. The virtual absence of proteome-based genome annotation for rice is possibly due to the absence of accurate and detailed rice proteome information.

Here we present (OryzaPG-DB) a rice proteome database based on shotgun proteogenomics. Unlike the currently available rice proteome database [27], which provides the 2D-PAGE-based proteome, OryzaPG-DB contains peptides obtained from shotgun-based proteomics with their product ion spectra, as well as updated annotations, side by side with the corresponding protein, cDNA, transcript and genomic sequences and information.

\section{Construction and content}

Generation of a reference dataset by shotgun proteomics

To perform proteogenomics-based genome annotation refinement of rice, we firstly generated a dataset by using a shotgun proteomics approach with an LTQ-orbitrap highaccuracy mass spectrometer for analysis of undifferentiated cultured rice cells (see supplementary methods in additional file 1). The experimental workflow is shown in Figure 1. Three pre-fractionation procedures, SDS-PAGE at the protein level, and strong cation exchange chromatography (7 runs) [28] and isoelectric focusing (7 runs) [29] at the peptide level, were employed prior to nanoLC-MS/ MS analysis to extend the proteome coverage. As a result, 156,871 product ion spectra were acquired from 27 LCMS/MS runs. Undifferentiated cultured rice cells were selected as the first sample to construct our bioinformatics pipeline and data repository system.

Regarding the databases for MASCOT [30] search, the Rice Genome Annotation Project, currently at Michigan State University (MSU), offers rice protein, cDNA, transcript and genome databases (MSU DB) as de novo annotation of the rice genome sequence, with further improvements and modifications using full-length cDNA and EST alignment [26] Thus, we decided to use these four databases for peptide/protein identification, since each of them provides a special opportunity to identify novel peptides. The protein database provides all potential protein and peptide sequences. The cDNA database is similar to the protein database, but allows searching six-frame translations of the nucleotide sequence, and therefore, it is possible to identify exonexon junction peptides and exon-skipping events from the two databases [18,31]. The transcript database includes introns, so we can identify exon-intron spanning and intronic peptides. Finally, the genome database includes the intergenic regions, offering the potential to find new non-annotated genes. Thus, each of the four databases affords specific search advantages (Figure 2).

As a result, we identified 14,955 unique peptides from the database searching against MSU protein database (V6.1). Further, we identified 166 additional unique peptides from three nucleotide sequence databases (cDNA, transcript and genome databases). Then, the peptides obtained in this study were mapped to the annotated MSU genome (V6.1) to identify proteins (cDNAs) and unspliced mRNAs (genes) (Table 1). Genes with peptides mapped to novel regions such as intron, exonintron boundary and non-coding region, are counted as "Genes to be revised", indicating that the annotation of these genes needs revision.

\section{Proteogenomics analysis to find novel genomic features} Next, we performed proteogenomic data analysis using bioinformatics approaches to map the identified peptides 


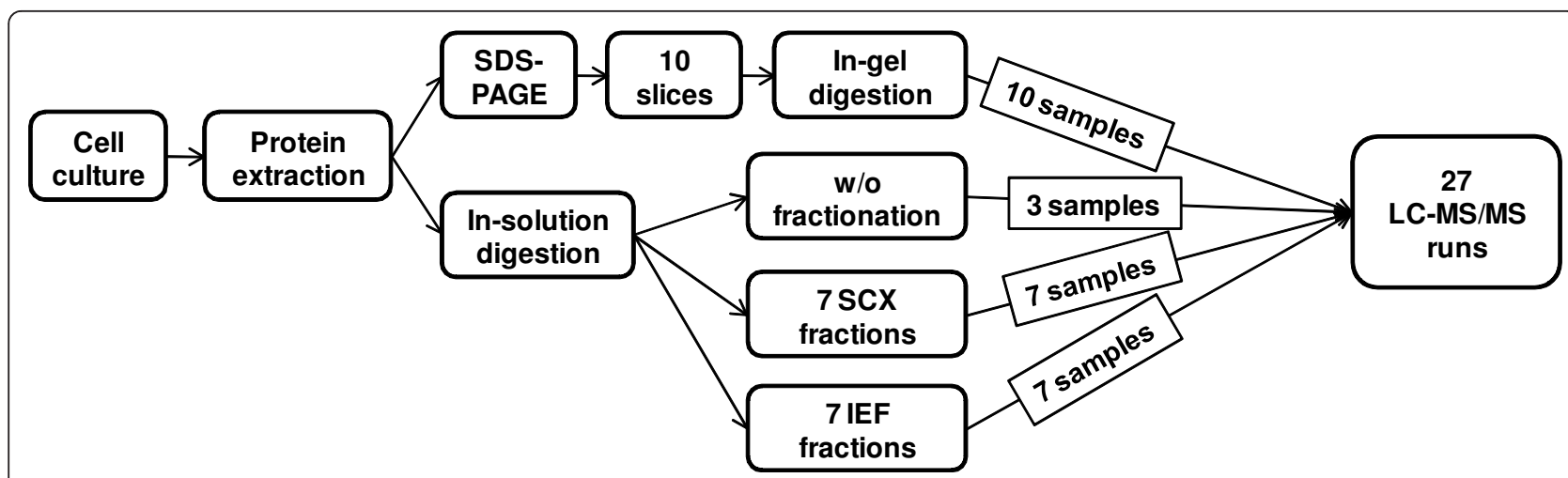

Figure 1 The experimental workflow to obtain the rice proteome. Proteins from undifferentiated cultured cells were extracted, digested, and pre-fractionated, and 27 samples were prepared for the subsequent LC-MS/MS analysis.

back to the genome and find novel genomic features as follows:

- Download the original annotation from MSU genome browser with only the MSU Osa1 Rice Gene Models and MSU Osa1 Rice Loci features selected. The original files can also be obtained from the OryzaPG-DB download page.

- Align all peptides identified from the MSU protein, cDNA and transcript databases to their corresponding genomic origin (genomic-unspliced mRNA), using the Basic Local Alignment Search Tool (BLAST) [32]. The alignments were performed using a local version of NCBI BLAST (blast2seq) [33] and perl script.

- Extract the alignment results of the peptides identified from the MSU genome database directly from MASCOT output files.
- Create perl scripts that read the alignment results and convert them to standard GFF3 format. Each peptide's alignment was converted to a GFF3 line indicating its type, identification source, start, end, parent and OryzaPG-DB peptide ID.

- Map the peptides identified from the MSU genome to the genes by comparing the peptide alignment coordinates (start and end) with the gene coordinates. If a peptide's start and end are between or overlapping with a gene's start and end, we map this peptide to that gene and create a GFF3 line similar to the one described above.

- Update the original annotation files by appending the peptides' GFF3 lines obtained from our analysis to the end of the corresponding gene. So far, we have created an updated annotation in GFF3 format containing

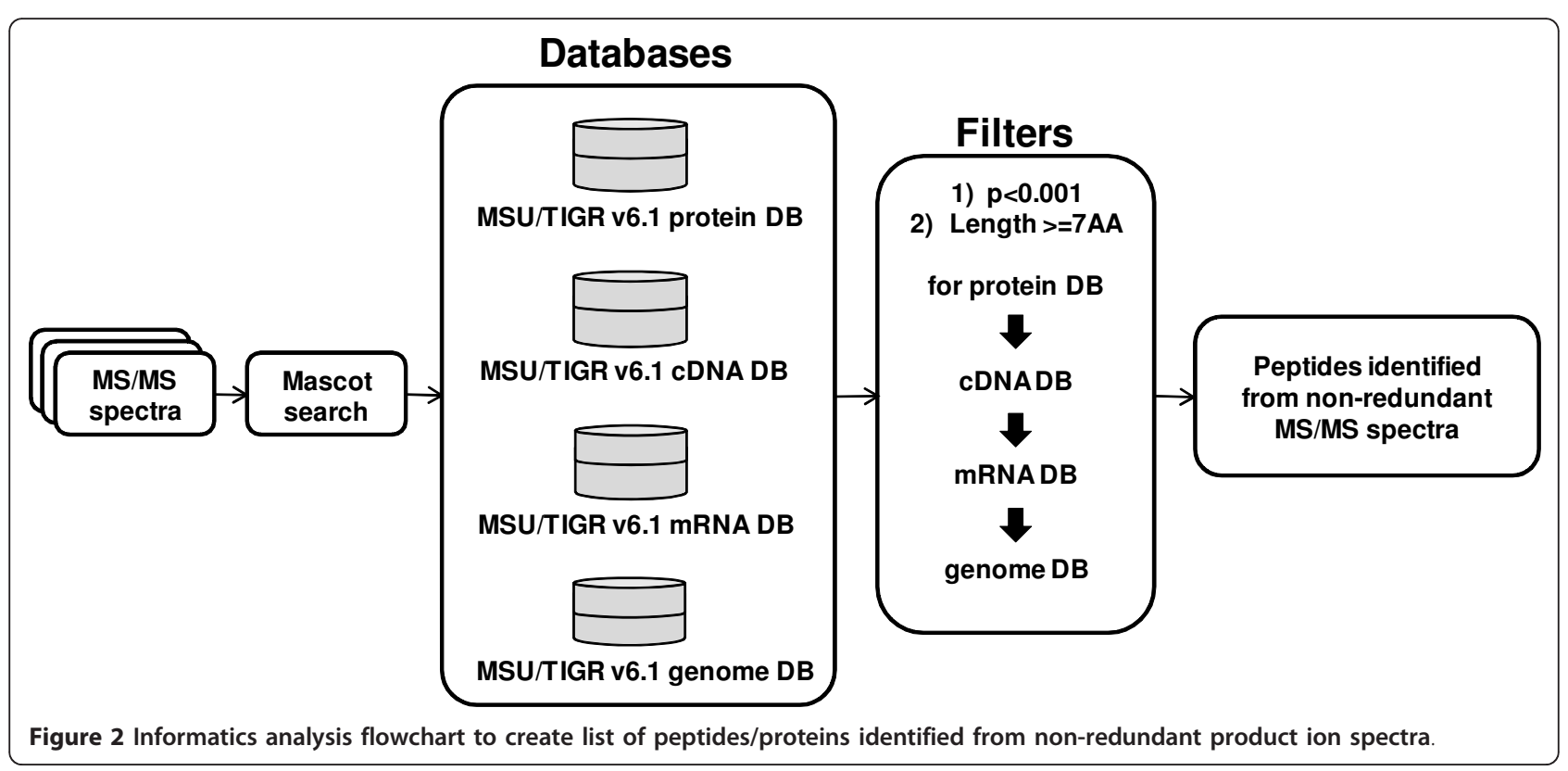


Table 1 Summary of the currently available contents in OryzaPG-DB*

\begin{tabular}{|c|c|c|}
\hline Item & $\begin{array}{l}\text { Current } \\
\text { contents** }\end{array}$ & Remarks \\
\hline Proteins/cDNAs & 5,034 & Corresponds to MSU/TIGR (6.1) models \\
\hline Genes/us-mRNAs & 3,182 & Corresponds to MSU/TIGR (6.1) locus/transcript (TU) \\
\hline Total peptides & 15,121 & All identified non-redundant peptides \\
\hline Novel peptides & 166 & Peptides that are not present in the protein database \\
\hline $\begin{array}{l}\text { Genes to be } \\
\text { revised }\end{array}$ & 40 & $\begin{array}{c}\text { Genes with peptides mapped to novel regions such as intron, exon-intron boundary and non-coding } \\
\text { regions }\end{array}$ \\
\hline
\end{tabular}

* Values in this table are statistics of the database contents on the launching date. All updates can be found in the UPDATES page in OryzaPG-DB website.

** On 22/Feb/2011.

the original annotation and the proteome information. Thus, the "Type" column in the updated GFF3 files includes the type "peptide" beside the original types (3'UTR, 5'UTR, CDS,...etc). However, the identified novel peptides require further analysis to find out whether or not they represent novel genomic features.

\section{Peptide novelty assessment and visualization of gene features}

The genomic features can be visualized using tools such as the generic genome browser (Gbrowse) or UCSC genome browser $[34,35]$, but determination of whether or not the peptide represents a novel genomic feature and the type of novelty, e.g., intronic or exon-boundary spanning, cannot be done with these tools. We consider a peptide novel if it does not exist in the protein database. Therefore, all the peptides identified from the other three databases are considered novel. However, this does not mean that such a peptide represents a novel genomic feature. The peptide may be aligned to a known coding region, but may not exist in the protein database, due to incompleteness [36,37]. Hence, we need an evaluation tool and algorithm to assess the genomic novelty of each novel peptide. Therefore, we developed PGFeval (ProteoGenomic Features Evaluator), an evaluation and visualization tool using perl and the GD library http://www.libgd.org, which evaluates the genomic novelty of each peptide and draws the whole gene model with graphical annotation that incorporates the genomic novelty of the peptides. PGFeval analyzes the updated annotation file in GFF3 format and uses the type, start and end to draw the gene and its structural elements, such as the UTRs and exons (Figure 3A). Next, it implements an assessment algorithm to evaluate and cluster the peptides into four clusters (intronic, exon acceptor spanning, exon donor spanning and known) (Figure 3B). The four clusters are illustrated in Figure $3 C$. In addition, PGFeval exports two CSV reports, a genes report and a peptides report, in a master-slave style. The genes report contains one entry per gene summarizing the gene's features such as total peptides, number of novel peptides and novel genomic features, while the peptide report contains one entry per peptide, indicating its gene and assessment result, such as novelty, cluster and identification source. Thus, the two reports can be easily analyzed using any spreadsheet software or imported into any relational database as two tables with one-to-many relationship. Figure 3D shows an example of the PGFeval graphical output.

This analysis revealed 51 new genomic features in 40 genes. The majority of the novel features consisted of intronic peptides (36), while the exon boundary-spanning peptides consisted of 13 donor-spanning and 2 acceptor-spanning peptides. The remaining novel peptides were mapped to known coding regions.

\section{Generic scheme design for the relational database}

We attempt to design a generic and simple database scheme that would be suitable for such proteogenomic data and that could be used in similar shotgun proteogenomics projects. As long as the annotation main unit is the gene, in our design (Figure 4), the database main entity is the gene as well. We used the MSU V6.1 locus as our gene ID, joining all other gene components together. All other database entities, such as protein, cDNA, unspliced mRNA and peptide, take the gene ID as a foreign key. Further, protein, cDNA, and un-spliced mRNA have their own IDs and aliases that were used in MSU V6.1. Peptide IDs start with P, C, T or G, which indicates that the identification source of the peptide is the protein, cDNA, transcript or genome database, respectively. The letter is followed by the serial number of the peptide in its sample, e.g., P345 is the peptide number 345 among the peptides identified from the protein database. The peptide ID also joins the peptide with its MASCOT results page and product ion spectral details. In the updated annotation files, the peptides are assigned to their parents using the notation "parent ID: peptide ID” e.g. LOC_Os06g01230.1:P62531.

\section{Database implementation and web interface development}

As mentioned above, PGFeval exports two reports: genes report and peptides report. Both reports are designed in 


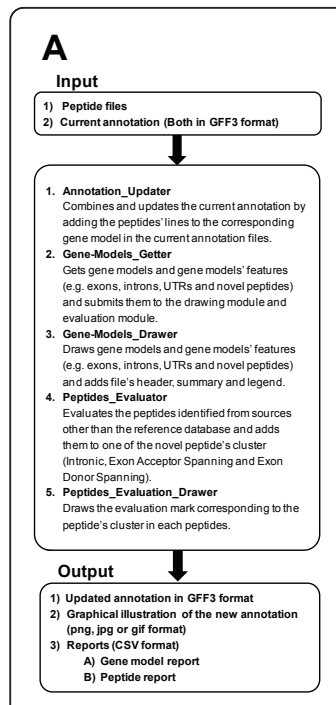

\section{B}

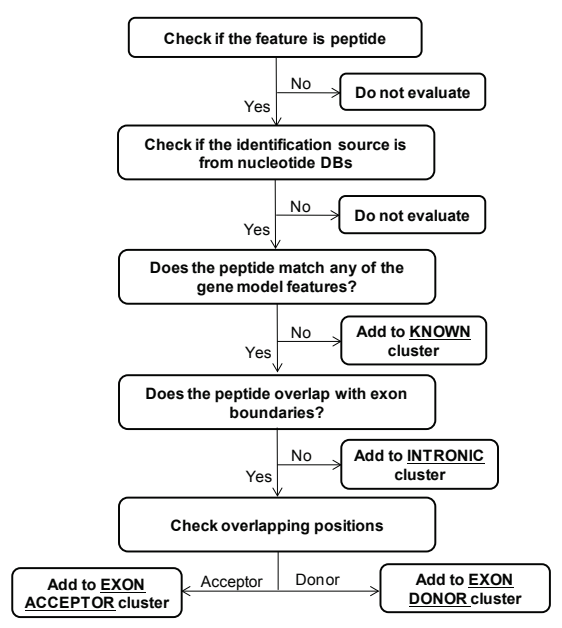

C

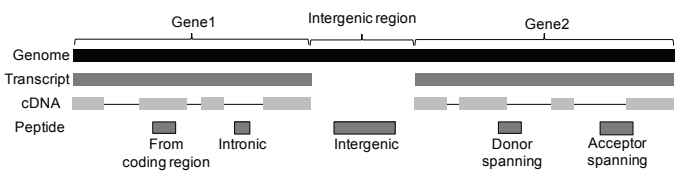

D Annotation and Proteogenomic Features of LOC_Os01g01307 Gene Annotation Visualization and Feature Assessment by PGFeval v1.1

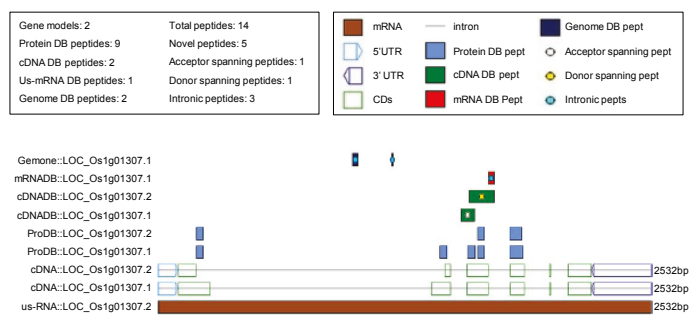

Figure 3 Assessment and visualization of a peptide's genomic novelty. (A) The design and architecture of PGFeval. (B) The assessment algorithm used in evaluating the peptide's novelty in PGFeval. (C) Schematic illustration of peptide clusters. (D) Example of the graphical output of PGFeval.

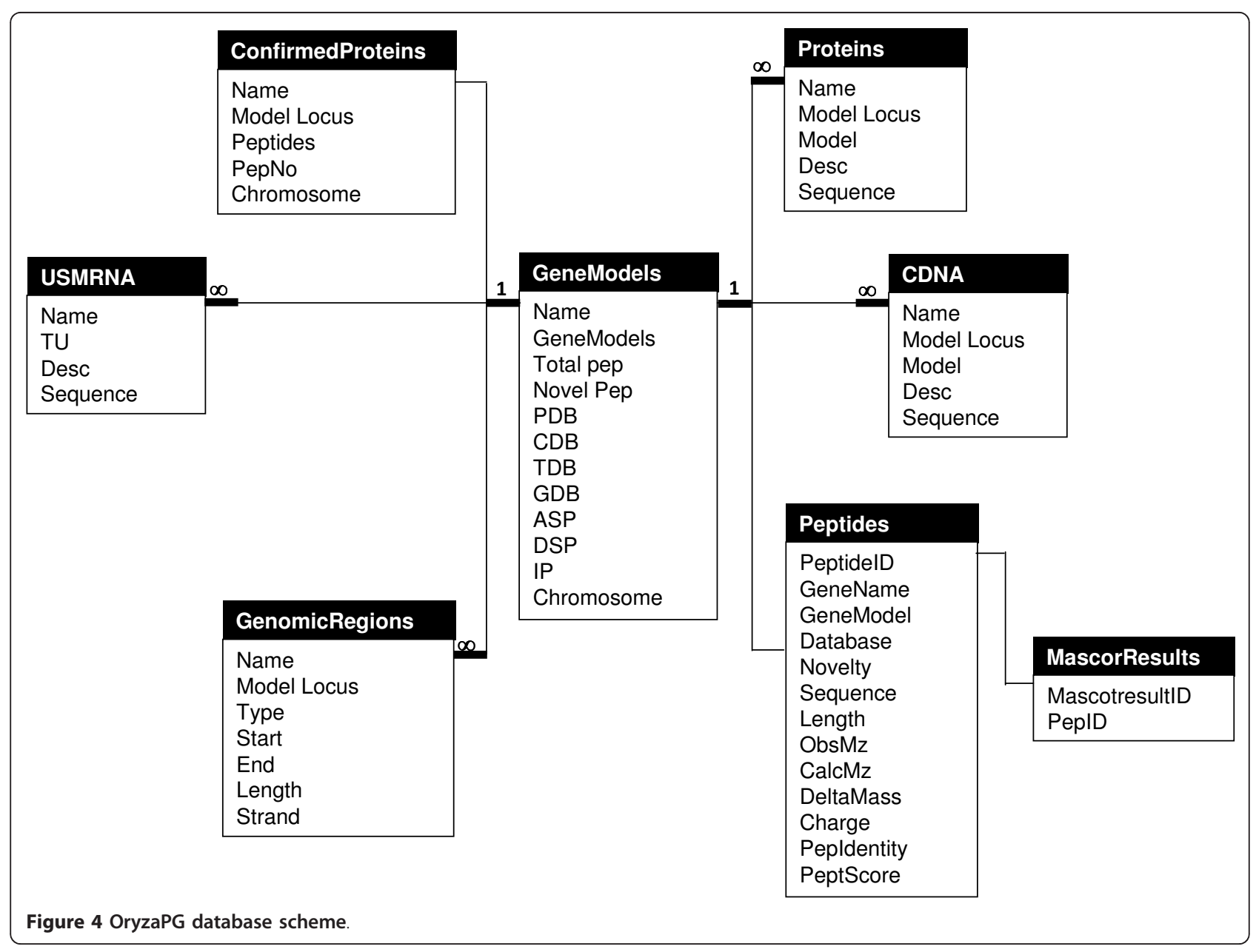


master-slave style. Thus, both were imported directly into the database. The protein, cDNA, and transcript information such as the IDs, aliases, descriptions, lengths and sequences were extracted from the FASTA files and the GFF3 files obtained from the MSU website and MSU genome browser, then converted to tables using perl scripts. Next, HTML files, similar to MASCOT peptide view files, were generated and imported into the OryzaPG-DB server. The data were later imported into a database implemented using the MySQL server. The annotation files (GFF3) and the visualization files (PNG) are stored in the web server directly. The whole system is thus a two-tier web-based system.

The web interface was developed using HTML, Java script and the server side scripting was done using PHP. The database was implemented using the MySQL server. We host the system on Microsoft Internet Information Service (IIS V7.5) on a Dell server running Windows 7 at the Institute for Advanced Biosciences (IAB), Keio University.

\section{OryzaPG-DB Application Programming Interfaces (APIs)}

The application programming interface (API) is an interface implemented by the application to allow interaction with the operating system or other programs. An API determines the protocol and parameters required to run certain functions or parts of the program and to return the results of its execution [38].

In OryzaPG-DB, we provide users with several URL APIs for data retrieval. For each entity, we provide users with an API that returns the results per record, per chromosome or for the whole genome. The data are returned in tabular view or in FASTA format with minimum formatting to allow easy processing. The complete list of the available APIs and their parameters can be found on OryzaPG-DB API Guide, available in the OryzaPG-DB website.

\section{Utility and Discussion}

\section{Shotgun proteogenomics}

The current rice genome annotation includes 56,797 genes, of which most are either putative (23,348 genes) or hypothetical $(8,885$ genes) or conserved hypothetical (2,003 genes) [26], http://rice.plantbiology.msu.edu]. Thus, the total number of genes for which experimental expression evidence is lacking represents more than $60 \%$ of the total annotated genes. Moreover, the available expression evidence (for 6,311 genes, representing about $10 \%$ of the total) is based on transcription, which does not necessarily imply translation to protein [8]. This indicates the need for a novel approach to improve and refine the rice genome annotation.

To perform genome annotation refinement of rice by means of a proteogenomics approach, we firstly need accurate and high-throughput proteome information. Thus, we generated the rice MS/MS-based proteome using our highly accurate nanoLC-MS/MS proteomics facility (see additional file 1). We started with undifferentiated cultured rice cells to generate data for the construction of our bioinformatics pipeline and data repository system, because a relatively unbiased expression profile of the rice proteome was expected, based on the report that an Arabidopsis thaliana proteomics study using cultured cells covered over $70 \%$ of the differentiated organ proteome [21]. We plan to generate similar datasets for all vegetative organs throughout the rice life cycle (see future work).

The generated data were compared against four databases (protein, cDNA, transcript and genome) for peptide/protein identification and the resultant peptides were filtered using Mascot score and peptide length to select peptides with high identification confidence and high specificity ( $\mathrm{p}$ value $<0.001$ and false-positive rate $(F P R)<=1 \%)$. Then, the peptides identified from the protein database together with the novel peptides identified uniquely from the other three databases were used to create list of peptides identified from non-redundant product ion spectra.

We utilized these peptides to perform proteogenomic analysis for the rice genes within our sample coverage. Our analysis revealed novel genomic features in 40 genes. In addition, 112 peptides, from the genome database-identified peptides, were mapped to intergenic regions, indicating the possible existence of non-annotated genes.

\section{OryzaPG-DB Utility}

OryzaPG-DB provides the scientific community with the first high-throughput MS/MS-based rice proteome information and proteogenomic results, publicly available through a comprehensive web interface (Figure 5). The web interface provides several means of data acquisition. Users can browse the database displaying data for genes, updated gene models, proteins, cDNAs and transcripts for the whole genome or per chromosome. In each case, the details of each record will be displayed together with links to related products, genomic origin and identified peptides. In addition, the sequence in FASTA format, the annotation in GFF3 format and visualization in PNG are available for download per displayed record. Further, external links to other databases such as NCBI, MSU and RAP are available. Users can also search the database using keyword search or parameter search, and the search results are displayed per gene. The download page provides users with all the data generated in this project and the data used to perform the proteogenomic analysis. Links to the websites that contains the original datasets and instructions for 


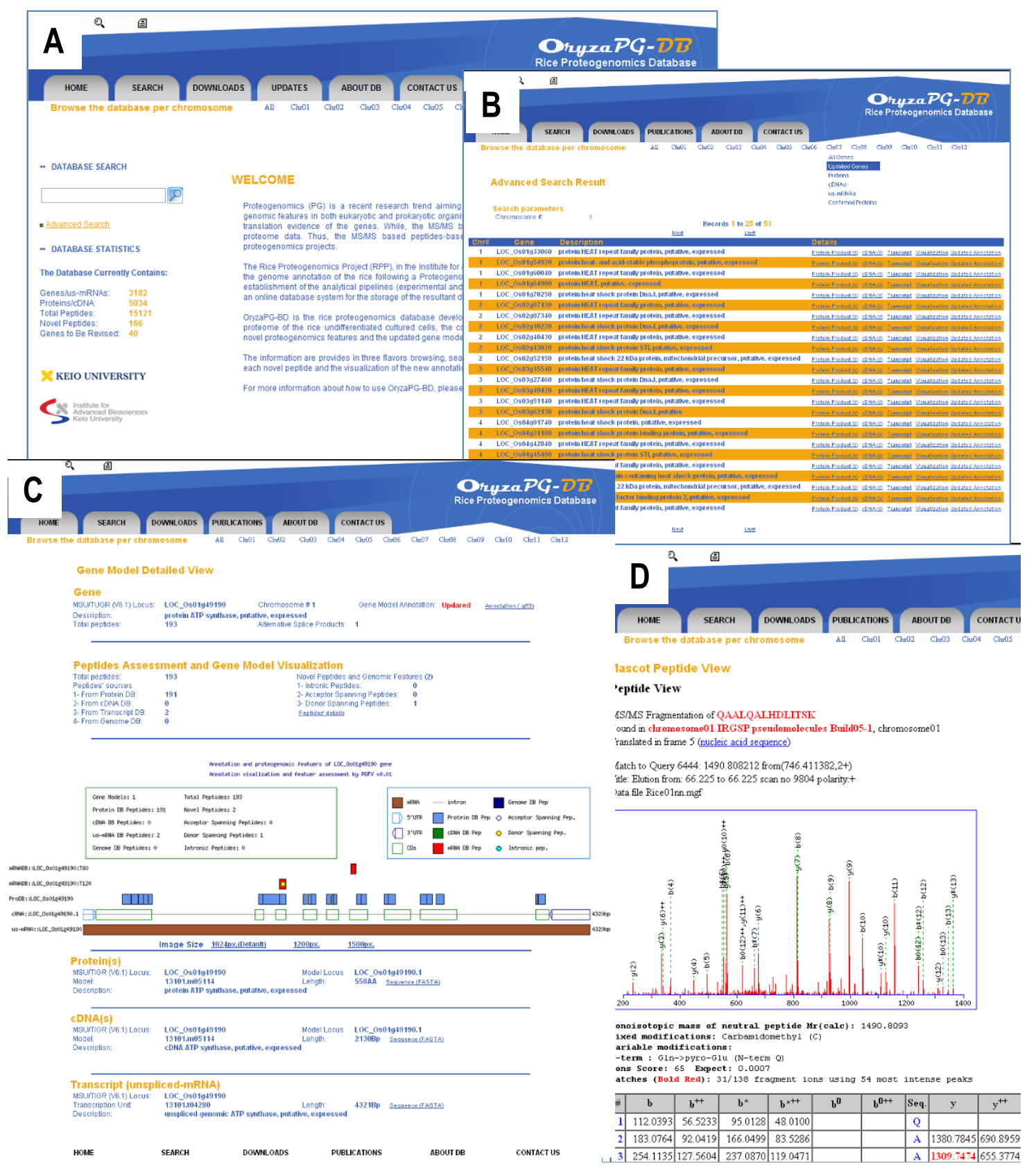

Figure 5 OryzaPG screenshots. (A) The advanced search form. (B) Example of advanced search results. (C) Gene detailed view including information on the gene, its protein products, CDNAs, transcripts and graphical visualization. D) Peptide view and the product ion spectra. The browse bar at the top of all pages allows the user to navigate through the database displaying data for all chromosomes or per chromosome.

how to download the original annotations are also provided.

\section{Future work}

Plans for further development of OryzaPG-DB are mainly focused on the content and consequently also the interface. We plan to extend the data to include rice root, stem, leaf blade and other organs as soon as we generate those proteomes. In addition, proteogenomic analysis will be available for all genes covered by the new samples. The interface, therefore, will be updated to allow browsing the data by sample, organ, etc., and we will also add advanced search parameters, enabling auto-generation of updated FASTA sequences using experimentally based genome reannotation. 


\section{Conclusions}

The rapid growth of available sequenced genomes requires novel approaches to identify genes and their functions, as well as sustainable data repository systems to store the accumulated data and make it publicly available for researchers. Proteogenomics is a novel approach combining MS/MS-based proteomics with genomic information and bioinformatics to enhance genome annotation. In this report, we present OryzaPG$\mathrm{DB}$, the data repository system of the Rice Proteogenomics Project. OryzaPG-DB provides interested rice biologists with the MS/MS-based proteome and the results of proteogenomic analysis, together with all the genomic information within our coverage. The database currently contains the results for cells from undifferentiated culture, and it is planned to be updated periodically with the results of analysis of samples from all vegetative organs of rice. We believe OryzaPG-DB will be an important resource and data-serving tool for rice biologists.

\section{Availability and Requirements}

OryzaPG-DB is freely available at http://oryzapg.iab.keio. ac.jp. In the development of Oryza-PG DB, we followed the usual standards of web applications development and the Java scripts employed are cross-browser scripts. We have confirmed that OryzaPG-DB is fully functional on four web-browsers, Google Chrome, Mozilla Firefox, Microsoft Internet Explorer and Safari, in five operating systems, Windows XP, Vista and 7, Linux Ubuntu and Mac OS (10.5), with no need for any plug-ins or special system requirements.

\section{Additional material}

Additional file 1: Supplementary Materials and Methods. Details of materials and experimental procedures used to produce the utilized dataset.

\section{Acknowledgements}

We would like to thank Professor Naoto Shibuya (Meiji University - Japan) and Dr. Hirofumi Nakagami (RIKEN Plant Science Center - Japan) for providing the rice cells, Dr. Nozomu Yachie (Harvard Medical School - USA), Dr. Naoyuki Sugiyama and Kalesh Sasidharan (Keio University - Japan) for valuable discussions, Yasuyuki lgarashi for technical assistance and other members of our institute for their contributions. This study is funded by Yamagata Prefecture and Tsuruoka City grants to Keio University, as well as by the Egyptian Bureau of Culture, Science and Education - Tokyo and The Global-Center of Excellence (G-COE) of Keio University to M. H. and JSPS Grants-in-Aid for Scientific Research (No. 21310129) and Science and Technology Incubation Program in Advanced Regions from Japan Science and Technology Agency to Y.I.

\section{Author details}

'Institute for Advanced Biosciences, Keio University, 403-1 Daihoji, Tsuruoka, Yamagata 997-0017, Japan. ${ }^{2}$ Systems Biology Program, Graduate School of Media and Governance, Keio University, 5322 Endo, Fujisawa, Kanagawa 252-
0882, Japan. ${ }^{3}$ Graduate School of Pharmaceutical Sciences, Kyoto University, Sakyo-ku, Kyoto 606-8501, Japan.

\section{Authors' contributions}

$\mathrm{Yl}$ designed the experiments and coordinated the whole project. $\mathrm{MH}$ performed the experiments, bioinformatics analysis, designed and developed PGFeval, the database and the web interface. MT supervised the project. MH wrote the first draft of the manuscript. All authors read and approved the final manuscript.

Received: 18 April 2010 Accepted: 12 April 2011

Published: 12 April 2011

\section{References}

1. Fullwood MJ, Wei CL, Liu ET, Ruan Y: Next-generation DNA sequencing of paired-end tags (PET) for transcriptome and genome analyses. Genome Research 2009, 19:521-532.

2. Siezen RJ, Hijum SAFTV: Genome (re-)annotation and open-source annotation pipelines. Microbial Biotechnology. Microbial Biotechnology 2010, 3:8.

3. Armengaud J: A perfect genome annotation is within reach with the proteomics and genomics alliance. Current Opinion Microbiolgy 2009, 12:292-300.

4. Koonin E, Galperin M: Sequence-Evolution-Function: Computational Approaches in Comparative Genomics. Kluwer Academic Publishers, USA; 2003.

5. Reed JL, Famili I, Thiele I, Palsson BO: Towards multidimensional genome annotation. Nature Reviews Genetics 2006, 7:130-141.

6. Wright JC, Sugden D, Francis-McIntyre S, Riba-Garcia I, Gaskell SJ, Grigoriev IV, Baker SE, Beynon RJ, Hubbard SJ: Exploiting proteomic data for genome annotation and gene model validation in Aspergillus niger. BMC Genomics 2009, 10:61.

7. Castellana NE, Payne SH, Shen Z, Stanke M, Bafna V, Briggs SP: Discovery and revision of Arabidopsis genes by proteogenomics. Proceedings of the National Academy of Sciences of the United States of America 2008, 105:21034-21038.

8. Ansong C, Purvine SO, Adkins JN, Lipton MS, Smith RD: Proteogenomics: needs and roles to be filled by proteomics in genome annotation. Briefings in Functional Genomics \& Proteomics 2008, 7:50-62.

9. Brent MR: Steady progress and recent breakthroughs in the accuracy of automated genome annotation. Natuar Reviews Genetics 2008, 9:62-73.

10. Coghlan A, Fiedler TJ, McKay SJ, Flicek P, Harris TW, Blasiar D, Stein LD: nGASP-the nematode genome annotation assessment project. BMC Bioinformatics 2008, 9:549.

11. Guigo R, Flicek P, Abril JF, Reymond A, Lagarde J, Denoeud F, Antonarakis S, Ashburner M, Bajic VB, Birney E, et al: EGASP: the human ENCODE Genome Annotation Assessment Project. Genome Biology 2006 7(Suppl 1):S2 1-31.

12. Allen JE, Pertea M, Salzberg SL: Computational gene prediction using multiple sources of evidence. Genome Research 2004, 14:142-148.

13. Castellana N, Bafna V: Proteogenomics to discover the full coding content of genomes: A computational perspective. Journal of Proteomics 2010, 73:2124-2135.

14. Armengaud J: Proteogenomics and systems biology: quest for the ultimate missing parts. Expert Reviews Proteomics 2010, 7:65-77.

15. Jaffe JD, Berg HC, Church GM: Proteogenomic mapping as a complementary method to perform genome annotation. Proteomics 2004, 4:59-77.

16. Wang R, Prince JT, Marcotte EM: Mass spectrometry of the M. smegmatis proteome: protein expression levels correlate with function, operons, and codon bias. Genome Research 2005, 15:1118-1126.

17. Tanner S, Shen Z, Ng J, Florea L, Guigo R, Briggs SP, Bafna V: Improving gene annotation using peptide mass spectrometry. Genome Research 2007, 17:231-239.

18. Power KA, McRedmond JP, de Stefani A, Gallagher WM, Gaora PO: Highthroughput proteomics detection of novel splice isoforms in human platelets. PLoS One 2009, 4:e5001.

19. Ansong C, Yoon H, Norbeck AD, Gustin JK, McDermott JE, Mottaz HM, Rue J, Adkins JN, Heffron F, Smith RD: Proteomics analysis of the causative agent of typhoid fever. Journal of Proteome Research 2008, 7:546-557. 
20. McCarthy FM, Bridges SM, Wang N, Magee GB, Williams WP, Luthe DS, Burgess SC: AgBase: a unified resource for functional analysis in agriculture. Nucleic Acids Research 2007, 35:D599-603.

21. Baerenfaller K, Grossmann J, Grobei MA, Hull R, Hirsch-Hoffmann M, Yalovsky S, Zimmermann P, Grossniklaus U, Gruissem W, Baginsky S: Genome-scale proteomics reveals Arabidopsis thaliana gene models and proteome dynamics. Science 2008, 320:938-941.

22. Sasaki T: Current status of and future prospects for genome analysis in rice. Springer-Verleg, Japan; 1999.

23. Matsumoto T, Wu J, Antonio BA, Sasaki T: Development in rice genome research based on accurate genome sequence. International Journal of Plant Genomics 2008, 2008:348621.

24. IRGSP: The map-based sequence of the rice genome. Nature 2005, 436:793-800

25. Itoh T, Tanaka T, Barrero RA, Yamasaki C, Fujii Y, Hilton PB, Antonio BA, Aono H, Apweiler R, Bruskiewich R, et al: Curated genome annotation of Oryza sativa ssp. japonica and comparative genome analysis with Arabidopsis thaliana. Genome Research 2007, 17:175-183

26. Ouyang S, Zhu W, Hamilton J, Lin H, Campbell M, Childs K, ThibaudNissen F, Malek RL, Lee Y, Zheng L, et al: The TIGR Rice Genome Annotation Resource: improvements and new features. Nucleic Acids Research 2007, 35:D883-887.

27. Komatsu S, Tanaka N: Rice proteome analysis: a step toward functional analysis of the rice genome. Proteomics 2005, 5:938-949.

28. Wu CC, MacCoss MJ, Howell KE, Yates JR: A method for the comprehensive proteomic analysis of membrane proteins. Nature Biotechnology 2003, 21:532-538.

29. Cargile BJ, Bundy JL, Freeman TW, Stephenson JL Jr: Gel based isoelectric focusing of peptides and the utility of isoelectric point in protein identification. Journal of Proteome Research 2004, 3:112-119.

30. Perkins DN, Pappin DJ, Creasy DM, Cottrell JS: Probability-based protein identification by searching sequence databases using mass spectrometry data. Electrophoresis 1999, 20:3551-3567.

31. Mo F, Hong X, Gao F, Du L, Wang J, Omenn GS, Lin B: A compatible exonexon junction database for the identification of exon skipping events using tandem mass spectrum data. BMC Bioinformatics 2008, 9:537.

32. Altschul SF, Gish W, Miller W, Myers EW, Lipman DJ: Basic local alignment search tool. Journal of Molecular Biology 1990, 215:403-410.

33. Tatusova TA, Madden TL: BLAST 2 Sequences, a new tool for comparing protein and nucleotide sequences. FEMS Microbiology Letters 1999, 174:247-250.

34. Stein LD, Mungall C, Shu S, Caudy M, Mangone M, Day A, Nickerson E, Stajich JE, Harris TW, Arva A, Lewis S: The generic genome browser: a building block for a model organism system database. Genome Research 2002, 12:1599-1610.

35. Karolchik D, Baertsch R, Diekhans M, Furey TS, Hinrichs A, Lu YT, Roskin KM, Schwartz M, Sugnet CW, Thomas DJ, et al: The UCSC Genome Browser Database. Nucleic Acids Research 2003, 31:51-54.

36. Bitton DA, Smith DL, Connolly Y, Scutt PJ, Miller CJ: An integrated massspectrometry pipeline identifies novel protein coding-regions in the human genome. PLoS One 2010, 5:e8949.

37. Fermin $D$, Allen BB, Blackwell TW, Menon R, Adamski M, Xu Y, Ulintz $P$, Omenn GS, States DJ: Novel gene and gene model detection using a whole genome open reading frame analysis in proteomics. Genome Biology 2006, 7:R35.

38. Tulach J: Practical API Design: Confessions of a Java Framework Architect. New York: Apress; 2008.

doi:10.1186/1471-2229-11-63

Cite this article as: Helmy et al:: OryzaPG-DB: Rice Proteome Database based on Shotgun Proteogenomics. BMC Plant Biology 2011 11:63.

\section{Submit your next manuscript to BioMed Central and take full advantage of:}

- Convenient online submission

- Thorough peer review

- No space constraints or color figure charges

- Immediate publication on acceptance

- Inclusion in PubMed, CAS, Scopus and Google Scholar

- Research which is freely available for redistribution

Submit your manuscript at www.biomedcentral.com/submit
Biomed Central 